\title{
Use of flow cytometry in the analysis of stage III squamous cell carcinoma of the oesophagus and its association with MIB-1
}

Department of Pathology, University of Hong Kong, Queen Mary Hospital, Pokfulam Road, Hong Kong

K Y Lam

$\mathrm{L} \mathrm{Ma}$

T F Luk

Department of Clinical Pathology, Pamela Youde Nethersole Eastern Hospital L K N Cheung

Department of Surgery, University of Hong Kong, Queen Mary Hospital J Wong

Correspondence to: Dr K Y Lam.

K Y Lam, Lily Ma, Simon Y K Law, Leslie K N Cheung, T F Luk, John Wong

\begin{abstract}
Aims-To examine the prognostic and pathobiological importance of DNA content in oesophageal squamous cell carcinomas in Hong Kong Chinese subjects; to evaluate its association with the immunohistochemical proliferative marker MIB-1.
\end{abstract}

Methods-Paraffin wax embedded tumour tissue and adjacent normal tissue (control tissue) samples from 45 resected stage III oesophageal squamous cell carcinomas were studied using flow cytometric analysis. The DNA content and the clinicopathological data of these patients were analysed together with the MIB-1 labelling index.

Results-DNA aneuploidy was present in $14(31 \%)$ of the 45 cases. However, the DNA content did not correlate significantly with the age, sex, or survival of the patients, nor the length, location, differentiation and MIB-1 labelling index of the oesophageal carcinomas. The synthetic (S) phase fraction of diploid tumours bore no relation to the patients' survival or MIB-1 score.

Conclusions-Flow cytometry was not as useful as the MIB-1 labelling index in predicting the biological characteristics of the tumours and the prognosis of patients with oesophageal squamous cell carcinomas. This study does not support the routine use of DNA flow cytometric analysis in oesophageal cancers.

( $F$ Clin Pathol 1996;49:975-978)

Keywords: flow cytometry, squamous cell carcinoma of the oesophagus, MIB-1 labelling index, prognosis.

An association between proliferative activity and overall prognosis has been noted in many cancers, and several studies have been published on the objective determination of cell proliferation using mainly either immunohistochemical methods or flow cytometry. ${ }^{12}$ We recently demonstrated the important role of the MIB-1 labelling index (using an immunohistochemical method) in relation to the grade of oesophageal squamous cell carcinoma and prognosis. ${ }^{3}$ Flow cytometric DNA analysis, on the other hand, has never been investigated in Hong Kong, although it is claimed to be more objective, precise, and quantitative (measuring large numbers of cells) than histological assessment, in the study of many cancers. ${ }^{4}$ The purpose of this study was to identify the correlation between the results of flow cytometry and a number of clinicopathological variables, including prognosis in Hong Kong Chinese patients with squamous cell carcinoma of the oesophagus, and to compare the efficacy of flow cytometry with the MIB-1 labelling index.

\section{Method}

Oesophageal tissues were collected prospectively from Chinese patients who had had oesophageal cancers treated by resection in Queen Mary Hospital, Hong Kong (a major referral centre for patients with oesophagea cancers) between January 1989 and December 1993. The samples from the oesophageal tumours and control tissue were obtained fresh, fixed in $10 \%$ formalin for less than 48 hours, processed and embedded in paraffin wax. In each case sections were cut and stained with haematoxylin and eosin and examined microscopically. The tumours were staged according to the tumour nodes metastases (TNM) classification. ${ }^{5}$ Tissue from patients with stage III squamous cell carcinomas were chosen for DNA measurement. One representative block from the tumour and one from the non-neoplastic tissue distal to the tumour were taken from each case for flow cytometric analysis. The tumour block used was the one in which the dominant histological pattern of each carcinoma was identified (because a few carcinomas showed slight variations in tumour differentiation in different foci of the tumours). The non-tumour blocks were processed simultaneously with the tumour blocks as an external check on instrument calibration, and as a control for specimen processing and staining.

The clinicopathological features of the cases were assessed. The age and sex of the patients as well as the site and the size (represented by the maximum longitudinal measurement) of the tumour were identified. The squamous carcinomas were graded into well, moderately, and poorly differentiated carcinomas according to World Health Organisation criteria. ${ }^{6}$ The 9 survival of the patients was measured from the date of resection to the dates of death or to the dates of last follow up.

Paraffin wax sections $(46 \mu \mathrm{m})$ from each chosen block were cut, dewaxed in xylene, and rehydrated in descending grades of alcohol and distilled water. The tissues were centrifuged at $1800 \mathrm{rpm}$ and the supernatant fluid poured off. The pellet in each case was digested in $1 \mathrm{ml}$ 
$0.5 \%$ pepsin (Sigma P-6887, USA) in $0.9 \%$ sodium chloride at $\mathrm{pH} 1.5$, and incubated in a water bath at $37^{\circ} \mathrm{C}$ with intermittent shaking for one hour. The suspension in each case was centrifuged at $1800 \mathrm{rpm}$ for five minutes. The supernatant fluid was poured off and the pellet was washed twice in phosphate buffered saline (PBS) and resuspended in $1 \mathrm{ml}$ PBS. The solution was filtered through nylon wool and the supernatant fluid removed by centrifugation, as before. The pellet was treated with equal parts $0.01 \%$ deoxyribonuclease free ribonuclease (Sigma R-5503, USA) and 0.01\% propoidium iodide (Sigma P-4170, USA) at $37^{\circ} \mathrm{C}$ and shaken for 30 minutes. The solution was then centrifuged, the supernatant fluid discarded, the pellet resuspended in PBS and the specimen taken for DNA measurement.

The DNA content was measured using a laser based, multiparameter flow cytometer (EPICS Profile II System, Coulter, Hialeah, Florida, USA), computed, and analysed using Multi-cycle Cell Cycle Analysis Software (Phoenix Flow Systems, San Diego, California, USA). More than 15000 cells were counted in each case. The specimens were classified into diploid and aneuploid categories according to their DNA index (DI) noted in the DNA histograms generated from the software. The samples with a DI of 0.9 to 1.1 were classified as diploid (fig 1) while those with a DI of more than 1.1 or less than 0.9 were classified as aneuploid (fig 2). Samples from the malignant tumours containing less than $20 \%$ tumour cells were considered unsuitable for evaluation and excluded from the study. The coefficient of variation $(C V)$ of the $G_{0} / G_{1}$ peak of each sample and the synthetic (S) phase fraction (the fraction of cells between the $G_{0} / G_{1}$ peak and the small $G_{2}+M$ peaks) of the diploid samples were also calculated from the histograms using the software. The CV is defined as the standard deviation (SD) divided by the mean. It is used to compare distributions and is often used as a measure of the quality of staining and instrument analysis. Samples were excluded from the study when the CV of the DNA $G_{0} / G_{1}$ diploid peak was $10 \%$ or greater.

The MIB-1 stained sections were counted at $\times 400$ magnification. At least 1000 nuclei were counted in each section, as described before. ${ }^{3}$ The MIB-1 score was presented as the number of positively stained nuclei per 1000 nuclei counted.

The results of clinicopathological assessment, survival analysis, DNA measurement and MIB-1 score of these cancers were computed. Statistical tests were performed using the SAS statistical package; test results were considered significant when a $p$ value of less than 0.05 was obtained.

Results

Forty five patients ( 37 men, eight women) with oesophageal cancer (13 well, 24 moderately, and eight poorly differentiated) were included in the study. The mean age of the patients was 64 years (range 47 to 78 years). The mean length of the lesions was $5.5 \mathrm{~cm}$ (range 1 to 11 $\mathrm{cm})$. The carcinomas were located in the upper

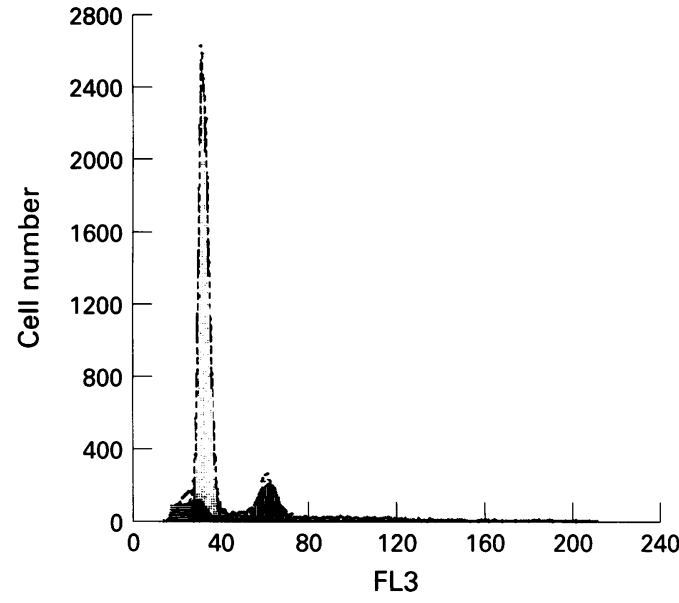

Figure 1 Histogram of DNA distribution: DNA diploid pattern $(D I=1)$. The diploid tumour has one distinctive $G_{o} / G_{1}$ peak and a tiny $G_{2}+M$ peak. The FL3 channel number of the $G_{2}+M$ peak is double that of the $G_{o} / G_{1}$ peak. (FL $3=$ relative fluorescence light intensity which represents the relative DNA content; $G_{0} / G_{1}, G_{2}+M=$ phases in the cell cycle).

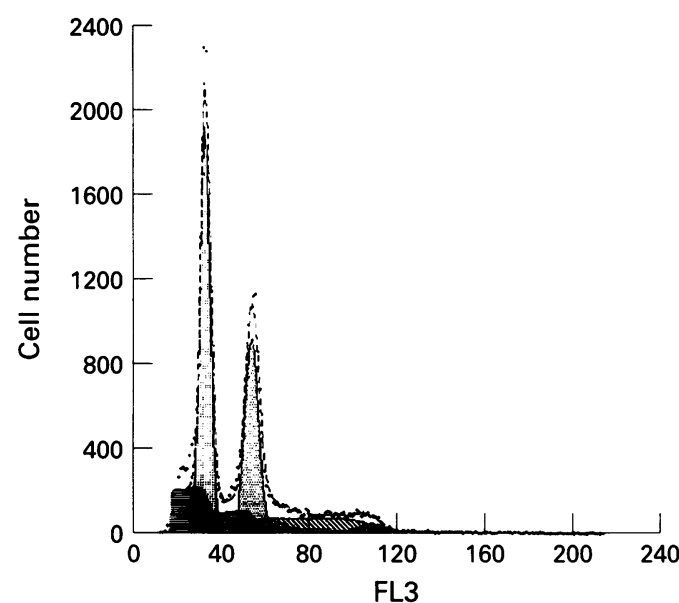

Figure 2 Histogram of DNA distribution: DNA aneuploid pattern $(D I=1.6)$. The aneuploid tumour has two distinctive $G_{o} / G$, peaks. The second peak comprises a substantial proportion of cells (much larger than the $G_{2}+M$ peak in the diploid pattern). The FL3 channel number of the second peak is $>1$ but is less than twice that of the first peak. (FL3 =relative fluorescence light intensity which represents the relative DNA content; $G_{o} / G_{1}, G_{2}+M=$ phases in the cell cycle).

portion of the oesophagus in four (9\%) patients, the central portion in $24(53 \%)$, and the lower portion in 17 (38\%). All follow up data from the patients were available and the mean follow up period for the whole group was 17 months. Forty (89\%) patients had died at the time of data analysis.

Sixty nine per cent ( 31 cases) of the tumours were diploid while $31 \%$ ( 14 cases) were aneuploid. The clinicopathological data of patients with diploid and aneuploid tumours are listed in table 1 . The DNA ploidy of the tumours did not correlate with the sex $(p=0.69$, Fisher's exact test) or age of the patients $(p=0.29$, $t$-test). Neither did it show any correlation with the longitude $(\mathrm{p}=0.58, t$-test $)$, location $\left(p=0.52, \chi^{2}\right.$ test) or MIB-1 score ( $p=0.73$, $t$-test) of the tumours. Although diploid tumours were more differentiated $(35 \%)$ than aneuploid tumours, there was no obvious difference in DNA content and histological grade $(p=0.073)$. 
Table 1 Clinicopathological data of squamous carcinomas of the oesophagus: aneuploid and diploid cases

\begin{tabular}{lll}
\hline Clinicopathological features & Aneuploid cases & Diploid cases \\
\hline No of patients & 14 & 31 \\
Average age & 65 & 63 \\
Male/female & $11 / 3(3: 1)$ & $26 / 5(5: 1)$ \\
Mean longitudinal length & $5.7 \mathrm{~cm}$ & $5.4 \mathrm{~cm}$ \\
Site: & & \\
$\quad$ Upper & $1(7 \%)$ & $3(10 \%)$ \\
$\quad$ Middle & $6(43 \%)$ & $18(58 \%)$ \\
Lower & $7(50 \%)$ & $10(32 \%)$ \\
Differentiation: & & \\
$\quad$ Well & $2(25 \%)$ & $11(35 \%)$ \\
Moderate & $7(58 \%)$ & $17(55 \%)$ \\
$\quad$ Poor & $5(17 \%)$ & $3(10 \%)$ \\
Mean MIB-1 score & 553 & 578 \\
\hline
\end{tabular}

The survival curves of the patients with diploid and aneuploid carcinomas are displayed in fig 3. Although patients with aneuploid stage III oesophageal squamous cell carcinomas seemed to have a slightly better prognosis, there was no significant difference in cumulative survival between the two groups $(p=0.23$, Wilcoxon test).

The $S$ phase could only be calculated for the diploid tumours. It ranged from $3.1 \%$ to $55.3 \%$. The resulting $\mathrm{S}$ phase value in these tumours bore no relation to either the survival of the patients $(\mathrm{p}=0.064$, Spearman correlation coefficients) or the MIB-1 labelling index $(\mathrm{p}=0.43$, Spearman correlation coefficients)

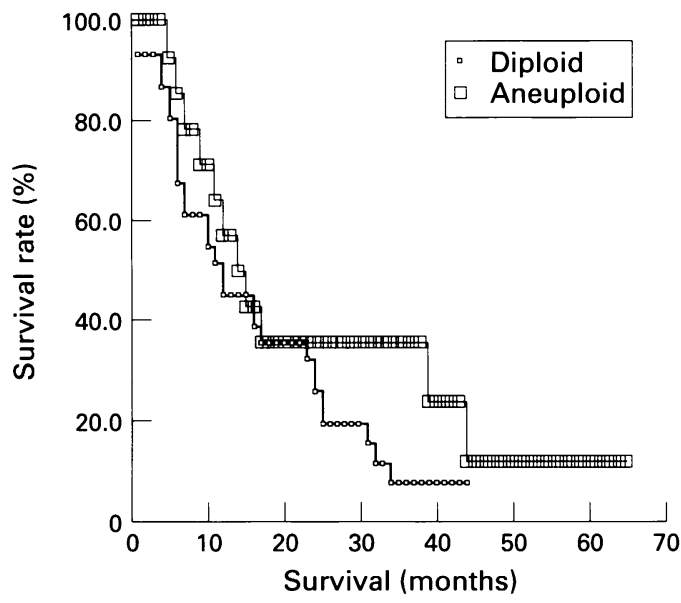

Figure 3 Survival of patients with diploid and aneuploid squamous cell carcinoma of the oesophagus.

\section{Discussion}

In this study an attempt was made to obtain more reliable results by limiting the value of an acceptable $C V$ of the $G_{0} / G_{1}$ peak to less than $10 \%$, counting a minimum of 15000 cells and using normal tissue in each case to act as a standard for staining and instrument control. We used paraffin wax sections because: (1) they are easier to obtain and handle than frozen tissues; (2) they can prevent contamination with a large number of normal cells by selecting the appropriate paraffin wax block with enough tumour tissue from the many blocks available. Only one frozen tumour block was available for each case and the quality of the section for interpretation was not as good as that found in paraffin wax sections; and (3) several studies have shown that equivalent results may be obtained from both paraffin wax and frozen sections in most instances, although significant variations occur among some laboratories. ${ }^{78}$

A review of the published series on flow cytometric analysis of DNA content in oesophageal squamous cell carcinomas is shown in table $2 .^{9-14}$ The prevalence of aneuploid tumours detected in these studies varied from $31 \%$ to $82 \%$. In these studies (including the present survey), DNA ploidy in oesophageal tumours was not related to either the age or sex of the patients nor to location or length of the tumours. ${ }^{911} 12$ The correlation between ploidy and grade in oesophageal squamous cell carcinomas has not been established. Besides, Yu et $a l^{9}$ and Doki et al ${ }^{11}$ reported that aneuploid tumours were less differentiated than diploid tumours, but in many other series (including this one), such differences have not been confirmed..$^{10-13} 20$

Yu $e t a l^{9}$ and Robey-Cafferty et $a l^{13}$ showed that aneuploid oesophageal tumours were often more advanced than diploid tumours, but other authors did not show similar findings. ${ }^{10131620}$ It is nevertheless worth noting that in our previous study, the prognosis of patients with oesophageal cancer depended strongly on the stage of the tumour. ${ }^{3}$ This study was limited to stage III oesophageal squamous cell carcinomas, to eliminate the effect of tumour stage on prognosis. In our experience most tumours were stage III at the time of surgery.

Table 2 Studies on flow cytometric analysis of DNA content in oesophageal squamous cell carcinomas

\begin{tabular}{lllllllll}
\hline Author (year) & Place & No & $\% A$ & $C V$ & Cell a & $T$ & Normal c & Remarks \\
\hline Yu $(1989)^{9}$ & China & 36 & 69 & $5 \%$ & 10000 & $\mathrm{~F}$ & Yes & \\
Edwards $(1989)^{10}$ & UK & 100 & 70 & $10 \%$ & 20000 & $\mathrm{P}$ & & \\
Katetani $(1989)^{11}$ & Japan & 31 & 74 & $10 \%$ & 20000 & $\mathrm{P}$ & & \\
Mannell $(1990)^{12}$ & South Africa & 42 & 79 & & 25000 & $\mathrm{P}$ & & \\
Robey-Cafferty & USA & 52 & 79 & & & $\mathrm{P}$ & & \\
$\quad(1991)^{13}$ & & & & & & & \\
Sasaki $(1991)^{14}$ & Japan & 23 & 87 & $3 \%$ & 10000 & $\mathrm{P}$ & & All at same stage (stage 2A) and \\
Dorman $(1992)^{15}$ & UK & 27 & 37 & $10 \%$ & 20000 & $\mathrm{P}$ & Yes & grade \\
& & & & & & $\mathrm{P}$ & & \\
Patil $(1993)^{16}$ & India & 74 & 81 & & 30000 & $\mathrm{P}$ & & \\
Doki $(1993)^{17}$ & Japan & 103 & 56 & & 33 & \\
Goukon $(1994)^{18}$ & Japan & 33 & 82 & & & $\mathrm{P}$ & & \\
Flejou $(1994)^{19}$ & France & 73 & 67 & $9 \%$ & & $\mathrm{~F}$ & & All superficial carcinomas \\
Haraguchi $(1995)^{20}$ & Japan & 40 & 71 & $8 \%$ & 10000 & $\mathrm{P}$ & & All stage 3 lesions \\
Lam (1996) & Hong Kong & 45 & 31 & $10 \%$ & 15000 & $\mathrm{P}$ & Yes &
\end{tabular}

$\%$ A: \% of aneuploid tumours; No: number of patients studied; CV: maximum value of coefficient of variation of $G_{0} / G_{1}$ phase accepted for analysis; T: type of tissue used; P: paraffin wax; F: frozen; Cell a: minimum number of cells analysed accepted for analysis; Normal c: presence of normal tissue from each patient to act as a control. 
Studies so far have shown conflicting results with regard to the value of DNA ploidy as a prognostic marker. ${ }^{21}$ In many solid tumours a diploid DNA pattern is usually associated with a favourable prognosis while DNA aneuploidy is associated with poor survival. In oesophageal cancers the relation between DNA ploidy and survival is even less clear. Of the few studies which have been performed, most have shown that flow cytometric DNA analysis was not useful in predicting patients' survival. ${ }^{12} 131516$ For instance, Mannell et $a l^{13}$ detected a higher recurrence rate while Doki et $a l^{17}$ demonstrated poorer survival in patients with aneuploid tumours. In contrast, Edward et al ${ }^{10}$ observed that if only tumours confined to submucosa or muscle wall (stage I/II tumours) were assessed, patients with diploid tumours had a poorer survival than those with aneuploid tumours.

In Hong Kong, we noted that there was no significant difference between the survival of patients with either diploid or aneuploid tumours, although diploid tumours seemed to have a slightly poor survival than aneuploid tumours. The association between DNA diploid lesions and poorer survival reported by Edwards $e t a l^{10}$ is actually not surprising as the explanation may be related to the theory that aneuploidy might disrupt or diminish rather than enhance the proliferation and spread of cancer cells. ${ }^{22}$

Both flow cytometric and immunohistochemical methods have been used in the determination of cell proliferation. ${ }^{12}$ In our previous study we documented the usefulness of the immunohistochemical stain, MIB-1, as an objective marker of tumour differentiation and a potential prognostic indicator in oesophageal squamous cell carcinoma. ${ }^{3}$ The current investigation, however, has failed to show any significant correlation between the $S$ phase fraction/ DNA ploidy and MIB-1. The counting of MIB-1 labelled nuclei seems to be better than flow cytometry in the objective assessment of the clinicopathological variables of oesophageal cancers.

In conclusion, flow cytometric analysis did not show any meaningful correlation with clinicopathological factors and prognosis. Flow cytometric analysis of DNA is also time consuming, depends heavily on technical expertise, and the initial cost of the flow cytometer is high. We therefore do not support the routine use of flow cytometry in the study of squamous cell carcinomas of the oesophagus in our population.

We thank Mr KH Yeung in the Department of Pathology and the technical staff in the Department of Surgery for their help.
1 Yu CCW, Filipe ML. Update on proliferation-associated antibodies applicable to formalin-fixed paraffin-embedded tissue and their clinical applications. Histochem $\mathcal{J}$ 1993;25:843-53.

2 Yu CCW, Woods AL, Levison DA. The assessment of cellular proliferation by immunohistochemistry: a review of currently available methods and their applications. Histochem $\mathcal{F}$ 1992;24:121-31.

3 Lam KY, Law SYK, So MKP, Fok M, Ma LT, Wong J Prognostic implication of proliferative markers MIB-1 and PC10 in oesophageal squamous cell carcinoma. Cancer 1996;77:7-13.

4 Carey FA. Measurement of nuclear DNA content in histological and cytological specimens: principles and applications. $\mathcal{F}$ Pathol 1994;172:307-12.

5 Bearhrs OH, Henson DE, Hutter RVP, Kennedy BJ Manual for staging of cancer. 4th edn. Philadelphia: JB Lippincott, 1992:57-9.

6 Watanabe H, Jass JR, Sobin LH, in collaboration with pathologists in eight countries. Definition and explanatory notes. In: International histological classification of tumours: histological typing of oesophageal and gastric tumours. 2nd edn. Berlin: Springer Verlag, 1990:11-8.

7 Hedley DW, Friedlander ML, Taylor IW, Rugg CA Musgrove EA. Method for analysis of cellular DNA content of paraffin-embedded pathological material using flow cytometry. $\mathcal{F}$ Histochem Cytochem 1983;31:1333-5.

8 Kumar D, Kumar S, Lyon D. Comparison of flow cytometric DNA content analysis of fresh and formalin-fixed paraffin-embedded solid neoplasms. Mod Pathol 1994;7: 354-60.

9 Yu JM, Yang LH, Guo Q, Chen YT, Li X, Zhong WX, et al. Flow cytometric analysis DNA content in oesophageal carcinoma: correlation with histologic and clinical features. Carcinoma: correlation

10 Edwards JM, Jones DJ, Wilkes SJL, Hillier VF, Hasleton PS Ploidy as a prognostic indicator in oesophageal squamous carcinoma and its relationship to various histological criteria. $\mathcal{F}$ Pathol 1989;159:35-41.

11 Kaketani K, Saito T, Kobayashi M. Flow cytometric analysis of nuclear DNA content in oesophageal cancer: aneuploidy as an index for highly malignant potential. Cancer 1989;64:887-91.

12 Mannell A, Hunter SJS, Becker PJ. DNA ploidy in oesophageal cancer: a preliminary report. South Afr $\mathcal{F}$ Sur 1990;28:55-8

13 Robey-Cafferty SS, El-Naggar AK, Sahin AA, Bruner JM Ro JY, Cleary KR. Prognostic factors in oesophageal squamous carcinoma: a study of histologic features, blood group expression, and DNA ploidy. Am 7 Clin Pathol 1991 95:844-9.

14 Sasaki K, Murakami T, Murakami T, Nakamura $M$ Intratumoral heterogeneity in DNA ploidy of oesophageal Intratumoral heterogeneity in DNA ploidy of oesoph

15 Dorman AM, Walsh TN, Droogan O, Curran B, Hourihance DOB, Hennessy TPJ, et al. DNA quantification of squamous cell carcinoma of the oesophagus by flow cytometry and cytophotometric image analysis usin formalin fixed paraffin embedded tissue. Cytometry 1992 13:886-92.

16 Patil P, Redkar A, Patel SG, Mistry RC, Deshpande RK Mittra I, et al. Prognosis of operable squamous cel Mittra I, et al. Prognosis of operable squamous cell patholgic features and DNA ploidy. Cancer 1993;72:20-4

17 Doki Y, Shiozaki H, Tahara H, Kobayashi K, Miyata M, Oka $\mathrm{H}$, et al. Prognostic value of DNA ploidy in squamous cel carcinoma of oesophagus: analysed wtih improved flow cytometric measurement. Cancer 1993;72:1813-8.

18 Goukon Y, Sasano H, Nishihira T, Nagura H, Mori S. p53 overexpression in human oesophageal carcinoma: a correlation with tumour DNA ploidy and two parameter flow cytometric study. Anticancer Res 1994;14:1305-12.

19 Flllejou JF, Muzeau F, Potet F, Lepelletier F, Fekete F, Henin D. Overexpression of the p53 tumour suppressors gene product in oesophageal and gastric carcinomas. Path Res Pract 1994;190:1141-8.

20 Haraguchi Y, Baba M, Takao S, Yoshinaka H, Hase S, Aikou $T$. Flow cytometric analysis of DNA heterogeneity in superficial carcinoma of the oesophagus. Cancer 1995;75: 914-9.

21 Koss LG, Czerniak B, Herz F, Wersto RP. Flow cytometric measurements of DNA and other cell components in measurements of DNA and other cell components in h28-48.

22 Rew DA. Significance of aneuploidy. Br $\mathcal{F}$ Surg 1994;81: 1416-2. 